\title{
Analysis of global terrorism dynamics by means of entropy and state space portrait
}

\section{António M. Lopes, J. A. Tenreiro Machado \& Maria Eugénia Mata}

\section{Nonlinear Dynamics}

An International Journal of Nonlinear Dynamics and Chaos in Engineering Systems

ISSN 0924-090X

Nonlinear Dyn

DOI 10.1007/s11071-016-2778-1

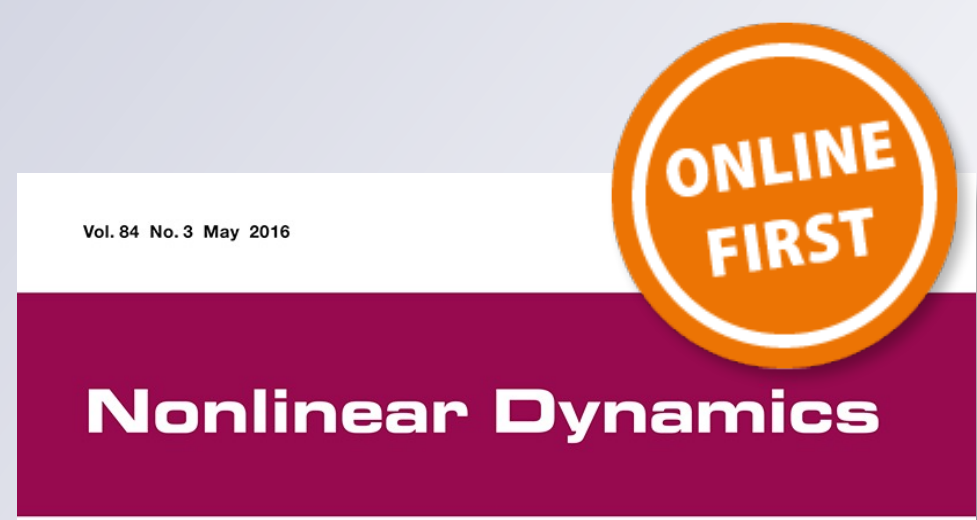

An International Journal of Nonlinear Dynamics and Chaos in Engineering Systems

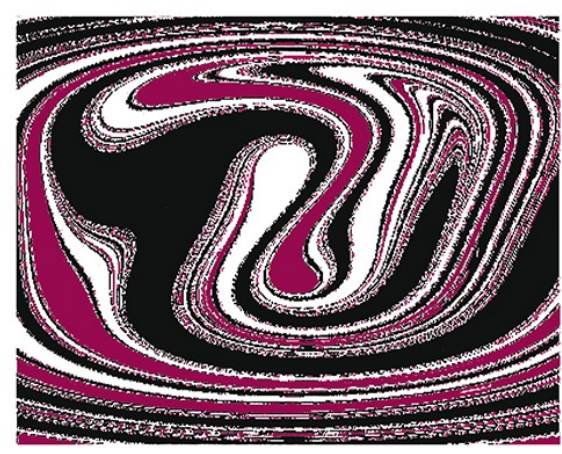

Springer 
Your article is protected by copyright and all rights are held exclusively by Springer Science +Business Media Dordrecht. This e-offprint is for personal use only and shall not be selfarchived in electronic repositories. If you wish to self-archive your article, please use the accepted manuscript version for posting on your own website. You may further deposit the accepted manuscript version in any repository, provided it is only made publicly available 12 months after official publication or later and provided acknowledgement is given to the original source of publication and a link is inserted to the published article on Springer's website. The link must be accompanied by the following text: "The final publication is available at link.springer.com". 


\title{
Analysis of global terrorism dynamics by means of entropy and state space portrait
}

\author{
António M. Lopes · J. A. Tenreiro Machado • \\ Maria Eugénia Mata
}

\begin{abstract}
This paper studies the global terrorism dynamics over the period 1970-2014. Data about terrorist events are analyzed by means of several mathematical tools, namely fractal dimension, entropy, state space portrait and multidimensional scaling, that reflect the dynamics in time and space. In a first phase, we consider worldwide events and we unveil the space-time characteristics exhibited by the global terrorism statistics. In a second phase, we group the events into eight geographic regions, and we analyze terrorism dynamics in a regional perspective. Finally, in a third phase, we adopt a complementary analysis of global terrorism based on multidimensional scaling and clustering techniques. The proposed methodology reveals to support new directions for exploring terrorism data.
\end{abstract}

Keywords Dynamical systems · Entropy · Fractal dimension $\cdot$ State space portrait $\cdot$ Multidimensional scaling $\cdot$ Terrorism

\section{Introduction}

Terrorism has afflicted the humankind since long. Small groups made of a variety of individuals and can pursue actions to provoke tremendous waves of panic and fearing, whose consequences cannot be measured. Among the consequences one may infer political effects to respond to the challenge, financial consequences to organize police and military reactions to persecute the actors [9], sociological consequences dictated by losses (human life, property and quietness) and economic consequences because of possible effects resulting from the reallocation of resources [23]. Tourism flow biases, industrial moves, brain drain outflows, emigration in general and remittances drops are good examples of consequences, as well as the demand for insurance for corporations and properties in general [7]. The twentieth century has been classified as the century of wars and crisis, but such a characterization is incomplete. It has also been a century of rising terrorism, a feature that translated to the new millennium. It is the purpose of this paper to access mathematical comprehensive models of behavior for this form of violence and disrespect for human rights. On the one side, grievances from groups (or regions) that are, or feel, weak may use terror actions to redress their ambitions 
and reach collective goals, in order to bring change to status quo situations they hate [48]. On the other side, terrorist attacks (usually using organized groups) can endure social cohesion in the attacked societies and motivate public opinions to ask new policies from government policy. If cohesion and political adjustments under pressure become accurate to persecute terrorism, short-run peaceful achievements will be reached, but exasperation of those who are or feel weak and used terror actions will motivate new waves of terror implementation in the long run.

Victory cannot be simply equated, unless those reasons that make those who are, or feel, weak may disappear. Ideological reasons (be they political, or religious), recruitment models, (radical) dogmas, technologies in use for the terrorist attacks or sociological cleavages behind terrorism devotion may have changed throughout time. However, and in spite of all these aspects, this paper contributes to go on applying regular mathematical techniques.

Bearing these ideas in mind, the paper is organized as follows. Section 2 describes the dataset used. Section 3 studies the time evolution of global terrorism by means of fractal geometry, entropy and space state portrait (SSP). Section 4 addresses terrorism dynamics in a regional perspective. Section 5 adopts multidimensional scaling (MDS) and clustering techniques for a complementary perspective on the terrorism dynamics. Finally, Sect. 6 draws the main conclusions and outlines the perspectives toward future work.

\section{Brief description of the dataset}

We use data from the global terrorism database (GTD). The GTD is available online and includes information on more than 140,000 worldwide terrorist attacks, from 1970 up to 2014 (http://www.start.umd.edu/gtd). Each record consists of the date of the event, geographic location (i.e., country, region, city and geographic coordinates) and total number of deaths and injuries, among other information.

We withdraw the data corresponding to year 1993, since for this year many events are missing. Moreover, we also discard all events with unknown geographic coordinates. The total number of valid records to be considered in the sequel is of 137,700 events.

The annual evolution of the number of occurrences and the geographic distribution of worldwide events

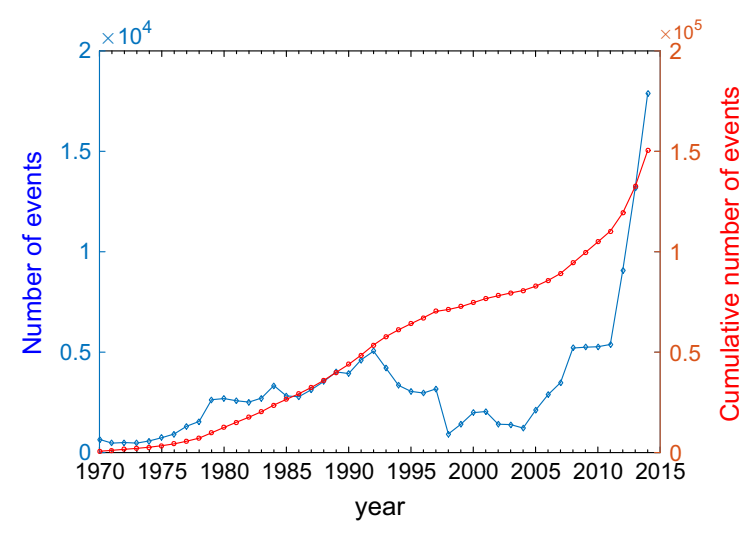

Fig. 1 Total number of terrorist events per year, during the period 1970-2014

during the period 1970-2014 are illustrated in Figs. 1 and 2 , respectively. It is visible an increasing trend in the terrorist activity until the beginning of the nineteens. Between 1990 and 2004 the number of events per year decreases. Since 2005 the number of attacks has raised quite fast. The regions of Middle East and North Africa, Sub-Saharan Africa, South Asia, Southeast Asia and the northwest region of the South America subcontinent have been the most affected.

\section{Analysis of global terrorism dynamics}

This section analyzes global terrorism by means of three mathematical tools that lead to complementary points of view. In Sects. 3.1 and 3.2 we study the fractal dimension of the spacial distribution and the entropy of worldwide events during the period 1970 2014. In Sect. 3.3 we adopt the SSP to visualize terrorism dynamics.

\subsection{Fractal dimension evolution of global terrorism}

A geometric fractal is an object composed of parts that are scaled versions of the whole [44]. This means that the same geometric pattern is observed, no matter the magnification that we use. Geometric self-similarity is ubiquitous in nature. In fact, many objects can be regarded as natural fractals, namely clouds, coastlines, snowflakes, crystals, blood veins and trees $[45,50]$. Fractals are usually characterized by statistical indices that measure the geometric complexity of an object, i.e., how the detail in a pattern changes with the scale 
Fig. 2 Geographic distribution of worldwide terrorist events during the period 1970-2014

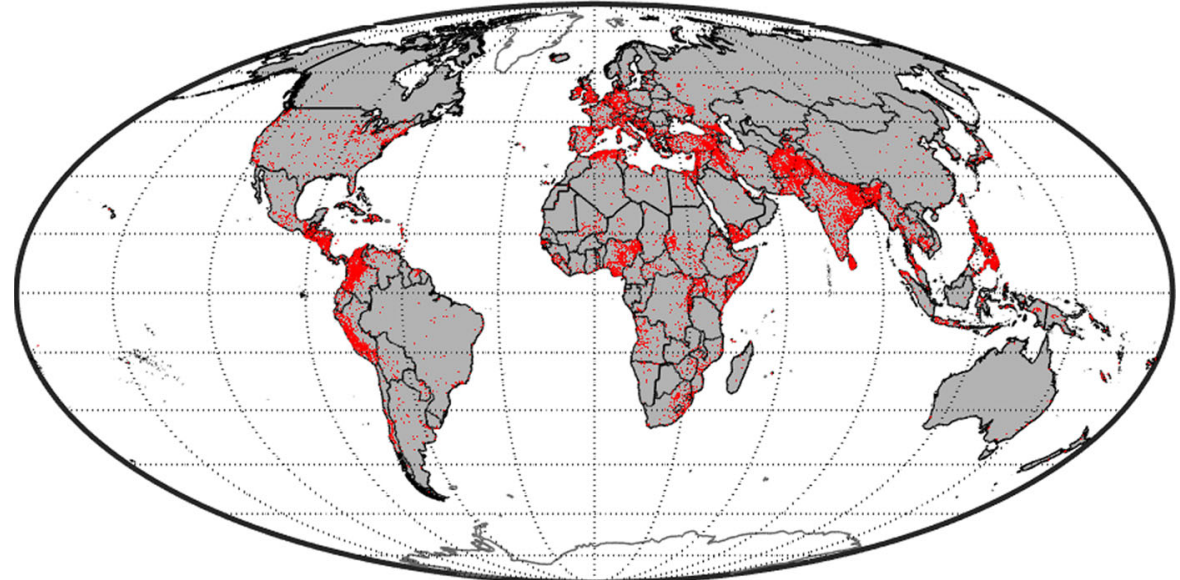

at which we observe it [36]. Self-similarity is related to the concept of scale invariance, which is mathematically described by power-law (PL) distributions [47,49,61]. Scale invariance has a statistical meaning, whereas self-similarity is a geometric concept [50]. The fractal dimension is a measure of how much the fractal fills the space as we zoom from larger to smaller scales, for which there are several definitions and, in general, do not coincide $[10,21,57]$. The definition more often found in practice is the box-counting fractal dimension, due to its easy numerical implementation. The algorithm can be described as follows:

- Repeat:

- cover the fractal object $S$ with a grid consisting of squares (the boxes) with size $\epsilon>0$,

- find the number of boxes, $N_{\epsilon}(S)$, that include part of the fractal,

- decrease $\epsilon$.

- The fractal dimension, $b$, corresponds to the slope of the log-log plot of $N_{\epsilon}(S)$ vs. $\epsilon$ :

$b(S)=-\lim _{\epsilon \rightarrow 0} \frac{\log N_{\epsilon}(S)}{\epsilon}$.

In other words, the fractal dimension is estimated as the exponent of a PL:

$y=a \epsilon^{b}, \quad\{a, b\} \in \mathbb{R}$.

For computing the fractal dimension, we project the geographic coordinates of the events in a Mercator cylindrical projection map and we apply the pro- cedure described above. The Mercator projection distorts shapes of large areas away from the equator, but with smaller effects on the latitude interval of interest (i.e., away from the northern and southern regions of Earth). The fractal dimension is in the interval $b \in[0.60,1.03]$, where the limits correspond to years 1972 and 2014, respectively. The value of $b$ for year 1993 is obtained by linear interpolation between adjacent values. Moreover, the time evolution of $b$ can be conveniently divided into eight periods, according to its behavior, that we will discuss in Sect. 3.2.

\subsection{Entropy evolution of global terrorism}

Entropy is a measure of complexity. In the last decades, generalizations of the classic BoltzmannGibbs-Shannon formulation have been proposed to explain the nature of complex systems that follow asymptotic PL statistical distributions [40]. These new entropic formulations are often expressed in terms of a set of parameters and, usually, do not obey the fourth Khinchin axiom [27]. Examples are the Tsallis [63], Rényi [55], Ubriaco [64], Kaniadakis [26], Sharma-Mittal [58] and Gamma [5] entropies, among others $[3,8,11,24,66]$. For particular values of the parameters, the classical entropic formulations are recovered.

The Boltzmann-Gibbs-Shannon entropy is given by:

$S=\sum_{i}\left(-\ln p_{i}\right) p_{i}$ 
and represents the expected value of the information content, $I\left(p_{i}\right)=-\ln p_{i}$, of an event with probability of occurrence $p_{i}$, where $\sum_{i} p_{i}=1$.

The Tsallis, $S_{q}$, Rényi, $S_{r}$, and Ubriaco, $S_{u}$, formulations are parametric entropies, given by:

$$
\begin{aligned}
& S_{q}=\frac{1}{q-1}\left(1-\sum_{i} p_{i}^{q}\right), \quad q \in \mathbb{R}, \\
& S_{r}=\frac{1}{1-r} \log \left(\sum_{i} p_{i}^{r}\right), \quad r \in \mathbb{R}^{+} \backslash\{1\}, \\
& S_{u}=\sum_{i}\left(-\log p_{i}\right)^{u} p_{i}, \quad u \in[0,1] .
\end{aligned}
$$

In the limit $\{q, r, u\} \rightarrow 1$, the Boltzmann-GibbsShannon entropy is obtained.

Fractional calculus (FC) deals with the generalization of integrals and derivatives to an arbitrary real, or complex, order $[28,46,56]$. FC models are able to capture long-range phenomena, often overlooked by standard differential equations, being an interesting tool for analyzing phenomena that occur in many dynamical systems with complex behavior $[6,25,31,32,37,38,42,43,60]$.

By adopting the tools of FC, the concepts of information content and entropy were recently revisited, leading to the information content and entropy of fractional order $\alpha \in \mathbb{R}[41,65]$ :

$$
\begin{aligned}
I_{\alpha}\left(p_{i}\right) & =D^{\alpha} I\left\{p_{i}\right\} \\
& =-\frac{p_{i}^{-\alpha}}{\Gamma(\alpha+1)}\left[\ln p_{i}+\psi(1)-\psi(1-\alpha)\right],
\end{aligned}
$$

$S_{\alpha}=\sum_{i}\left\{-\frac{p_{i}^{-\alpha}}{\Gamma(\alpha+1)}\left[\ln p_{i}+\psi(1)-\psi(1-\alpha)\right]\right\} p_{i}$,

where $D^{\alpha}\{\cdot\}$ is the derivative of order $\alpha$ and $\Gamma(\cdot)$ and $\psi(\cdot)$ represent the gamma and digamma functions, respectively.

The fractional (or generalized) entropy does not obey some of the Khinchin axioms except for $\alpha=0$ [41]. In this case it yields the classical BoltzmannGibbs-Shannon formulation (3). The entropy $S_{\alpha}$ was proven to be more sensitive to small variations in the statistical distributions of real-world phenomena $[35,39]$.
For calculating the entropy of the global terrorism attacks, we project the geographic coordinates of the events using a Mercator cylindrical projection and we estimate the probabilities according to the following procedure. First, we superimpose on the Earth's map a rectangular grid with a $5^{\circ}$ step (value chosen so that a lower limit does not significantly change results), yielding a total of $N=36 \times 72$ cells. We then compute the probabilities by using $p_{i}=\frac{n_{i}}{n}$, where $n_{i}$ is the number of events in each cell $i$ of the grid, and $n$ is the total number of points in the $N$ cells. The entropy for year 1993 is obtained by linear interpolation between adjacent values.

We start by comparing the results obtained with different entropy formulations. Figure 3 depicts the variation of the fractional, Tsallis, Rény and Ubriaco space entropies versus time and versus the entropy parameters $\{\alpha, q, r, u\} \in[0.1,0.9]$, respectively. We see that the fractional entropy, $S_{\alpha}$, has a maximum for $\alpha \approx 0.7$. The Tsallis, Rényi and Ubriaco entropies are both monotonic in their parameters. Furthermore, $S_{\alpha}$ is more sensitive to changes in its parameter $\alpha$ and leads to better discrimination between entropy values. Experiments with other entropy formulations [20] led to similar results.

We now compare the time evolution of the Boltzmann-Gibbs-Shannon, $S$, and the fractional, $S_{\alpha}$ ( $\alpha=0.7$ ), entropies for the period 1970-2014. The results are depicted in Fig. 4 that also shows the relationship between entropy and fractal dimension $b$. We see that $S_{\alpha}$ amplifies details, improving the resolution of the entropy charts. Moreover, the seven time periods foreseen while observing the behavior of parameter $b$ have good correspondence with the time evolution of the entropy, in particular with $S_{\alpha}$.

\subsection{Visualization of global terrorism using SSP}

For a $k$-dimensional dynamical system $(k \in \mathbb{N})$, the set of all its possible states, each one represented by a unique point, corresponds to the system state space. As time evolves, we obtain sequences of points that describe trajectories. The set of trajectories builds the SSP representation of the dynamical evolution along time. For $k \leq 3$, the SSP can be depicted and the system behavior can be inferred from the corresponding graphical representation [33,51]. 


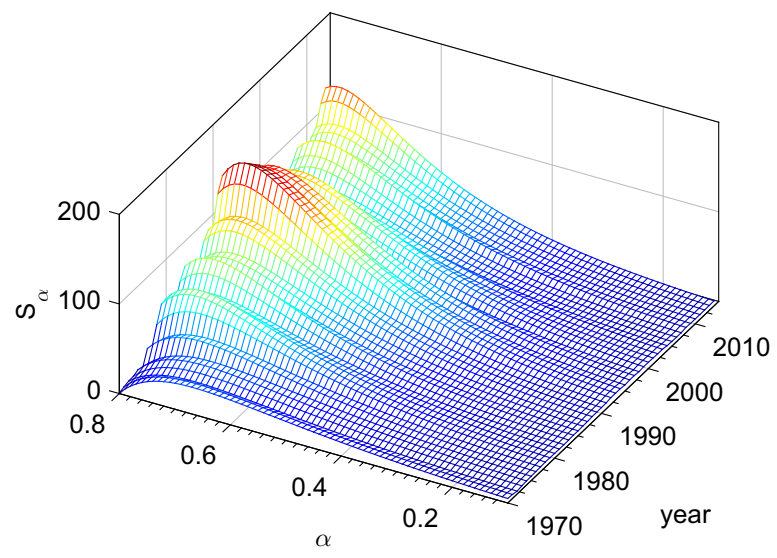

(a)

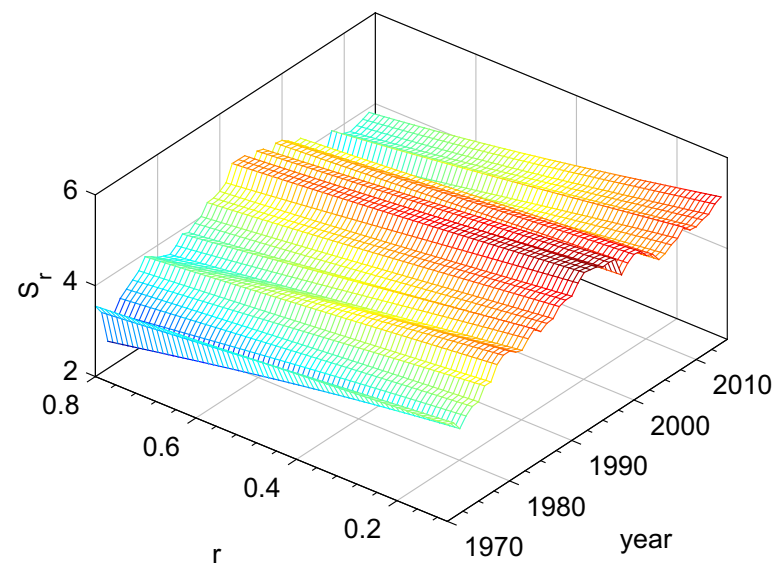

(c)

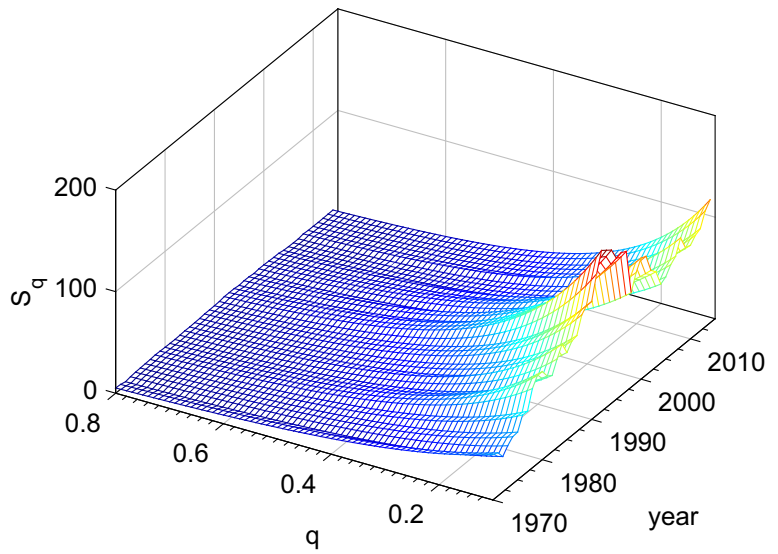

(b)

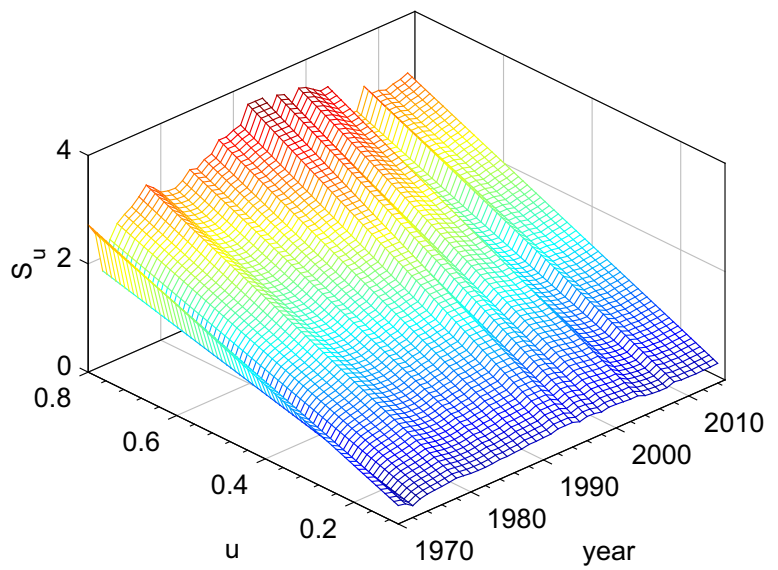

(d)

Fig. 3 Entropy of worldwide terrorism versus year and versus parameters $\{\alpha, q, r, u\} \in[0.1,0.8]$, for the period 1970-2014 and the entropic formulations: a fractional, $S_{\alpha} ; \mathbf{b}$ Tsallis, $S_{q} ; \mathbf{c}$ Rényi, $S_{r} ; \mathbf{d}$ Ubriaco, $S_{u}$

For constructing the SSP, we adopt as phase variables the time evolution of the entropy in the period 1970-2014 and its $k-1$ time derivatives. The model and the order of the system are unknown. Nevertheless, we tested successive increasing values of $k$ and found the 3-dimensional space to represent a good compromise between feasibility of the SSP representation and uniqueness of trajectory evolution by eliminating crossover points. The points corresponding to year 1993 are obtained by linear interpolation between adjacent values.

Figures 5 and 6 show the SSP representations for entropies $S$ and $S_{\alpha}(\alpha=0.7)$, respectively. The fractional entropy leads to a SSP representation where details can be identified easily and, as stated previously, are clearly correlated with the fractal dimension, $b$. From Figs. 4 and 6 we observe the following behavior:

- Period A: 1970-1977. Terrorist activity starts increasing in the second half of the 1970s of the last century. Since then, both the fractal dimension $b$ and entropy $S_{\alpha}$ growth moderately.

- Period B: 1978-1982 and Period C: 1983-1991. There is a growth trend in $b$ and $S_{\alpha}$. In $\mathcal{B}$ and $\mathcal{C}$ periods the parameters exhibit a similar behavior, yielding the loops observed in the SSP of Fig. 6.

- Period D: 1992-1997. Fractal dimension and entropy invert tendency. Then, we observe that both $b$ and $S_{\alpha}$ decrease to values close to those revealed in the previous decade. In the SSP we observe a 


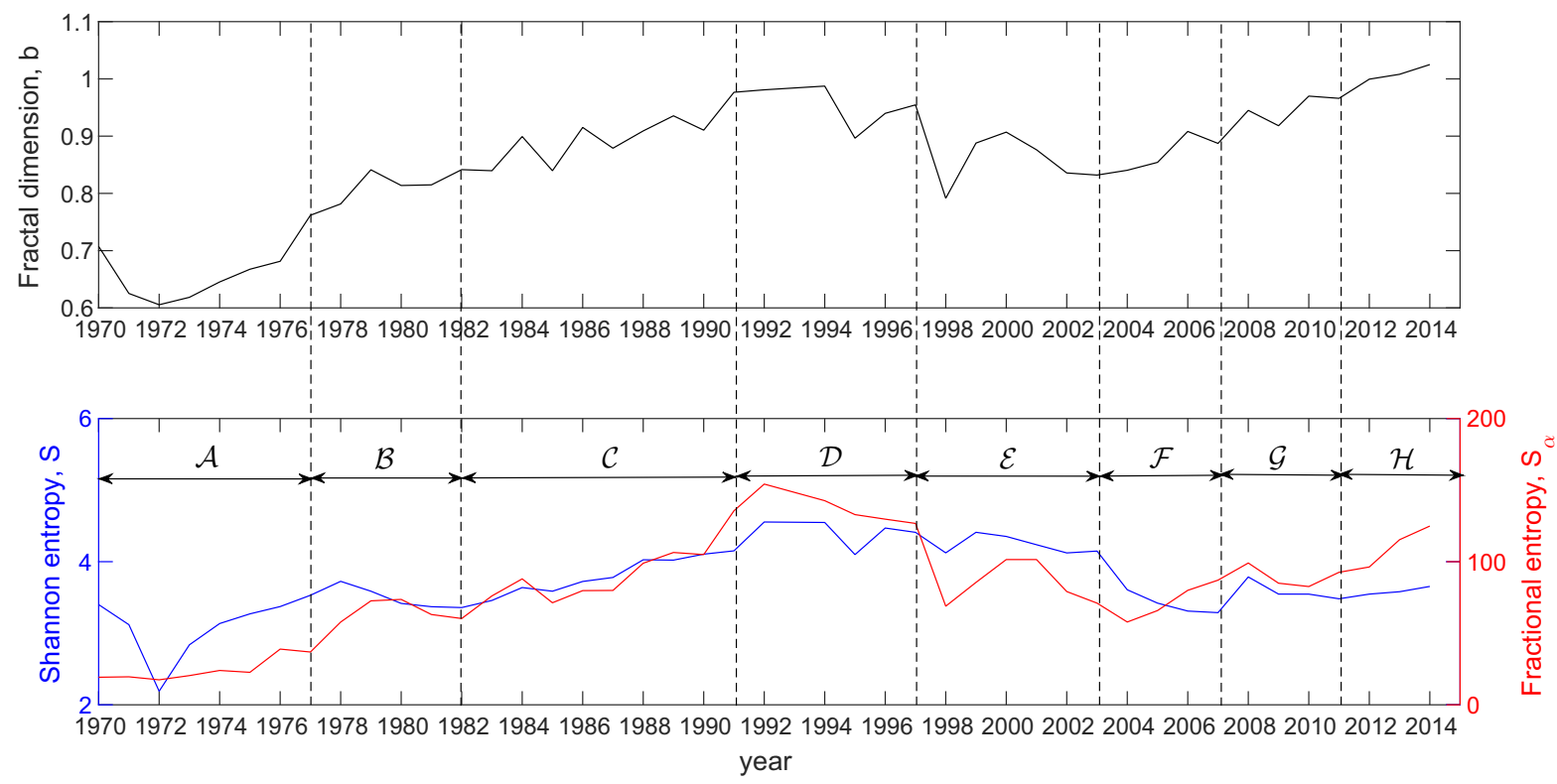

Fig. 4 Relationship between the fractal dimension, $b$, and the Boltzmann-Gibbs-Shannon, $S$, and fractional, $S_{\alpha}$, entropies, during the period 1970-2014

large loop connecting years 1991 and 1998, representing a fast transient.

- Period E: 1998-2003 and Period F : 2004-2007. In both periods $b$ and $S_{\alpha}$ exhibit a similar behavior, firstly rising up and afterward diminishing. Such behavior yields identical transient loops in the SSP.

- Period $\mathcal{G}:$ 2008-2011. There is stagnation period in $b$ and $S_{\alpha}$.

- Period $\mathcal{H}$ : 2012-2014. There is an increasing trend in $b$ and $S_{\alpha}$. Parameter $b$ reaches its maximum, while $S_{\alpha}$ approaches the values observed in year 1992. In the SSP we observe the beginning of what seems a large loop parallel to that of years 19911998.

At a different level, and looking at the SSP, we observe also the existence of two attractors, $\mathcal{A} \mathcal{T}_{1}$ and $\mathcal{A} \mathcal{T}_{2}$, more separated for $S$, and closer for $S_{\alpha}$. Does this means some kind of periodicity, or that conditions (and results) are similar or somehow repeating?

According to Weissman et al. [67] weak central governments and political regimes that are respectful to human rights tend to attract terrorism. Transfers of power to strong governments or political regimes that adopt death penalties may dissuade terrorist actions and explain historical loops. In spite of the difficulty that is involved in fighting terrorism, history provides good hopes in looking at political terrorism that could disappear thanks to victory. Political independences (from colonial situations), political autonomies and negotiated sharing power, for example, have provided large evidence on ending difficult terrorist situations, successfully. This is the case of the use of terrorism as a facet of colonial wars (against the Belgian, the French and the Portuguese colonial empires in Africa). This is also the case of the Irish Republican Army (IRA) pacification, the Basque terrorism pacification in Spain and the Colombian agreements on the long guerrillaterrorism events, for example [2]. Resilience on the one side of the conflict, against exhaustion on the other side of the conflict, has dictated the result and the end of terrorist attacks. They also can explain historical statistical loops. It is recognized that factors such as country income, unemployment and economic growth, social factors such as population, income disparity or political freedom, have a large effect on the number of terrorist events worldwide [18]. International support and geopolitical alliances (as well as their breakup) may give origin to terrorism loops [12]. The modern technologies of the new millennium have changed a lot the character of the terrorist attacks and their preparation. Computers and the cyber-crime, the control at distance for weaponry and the internet communication facilities are some of the aspects in use, in which experts 


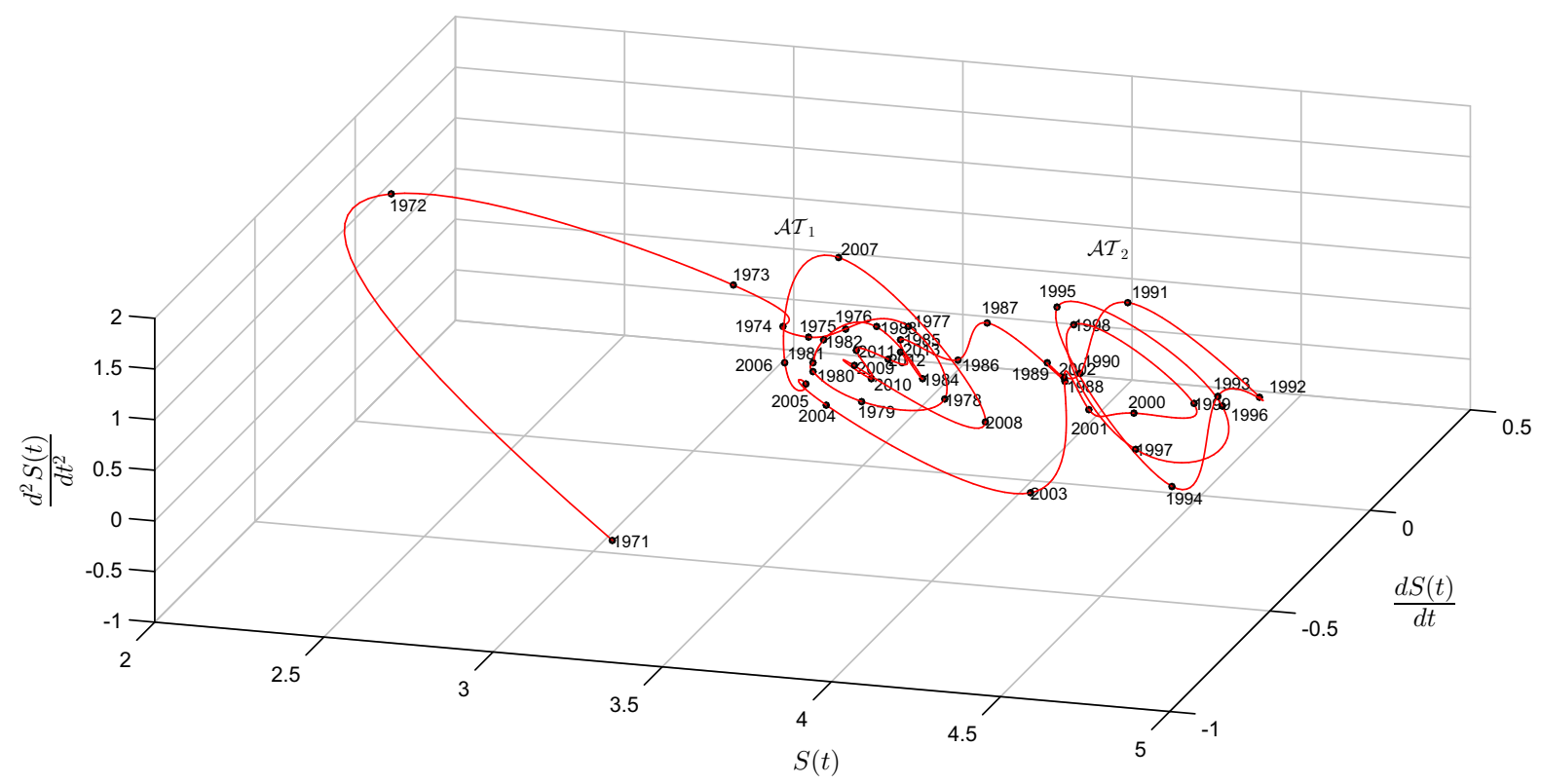

Fig. 5 The 3-dimensional SSP representation of $S$ of global terrorism dynamics

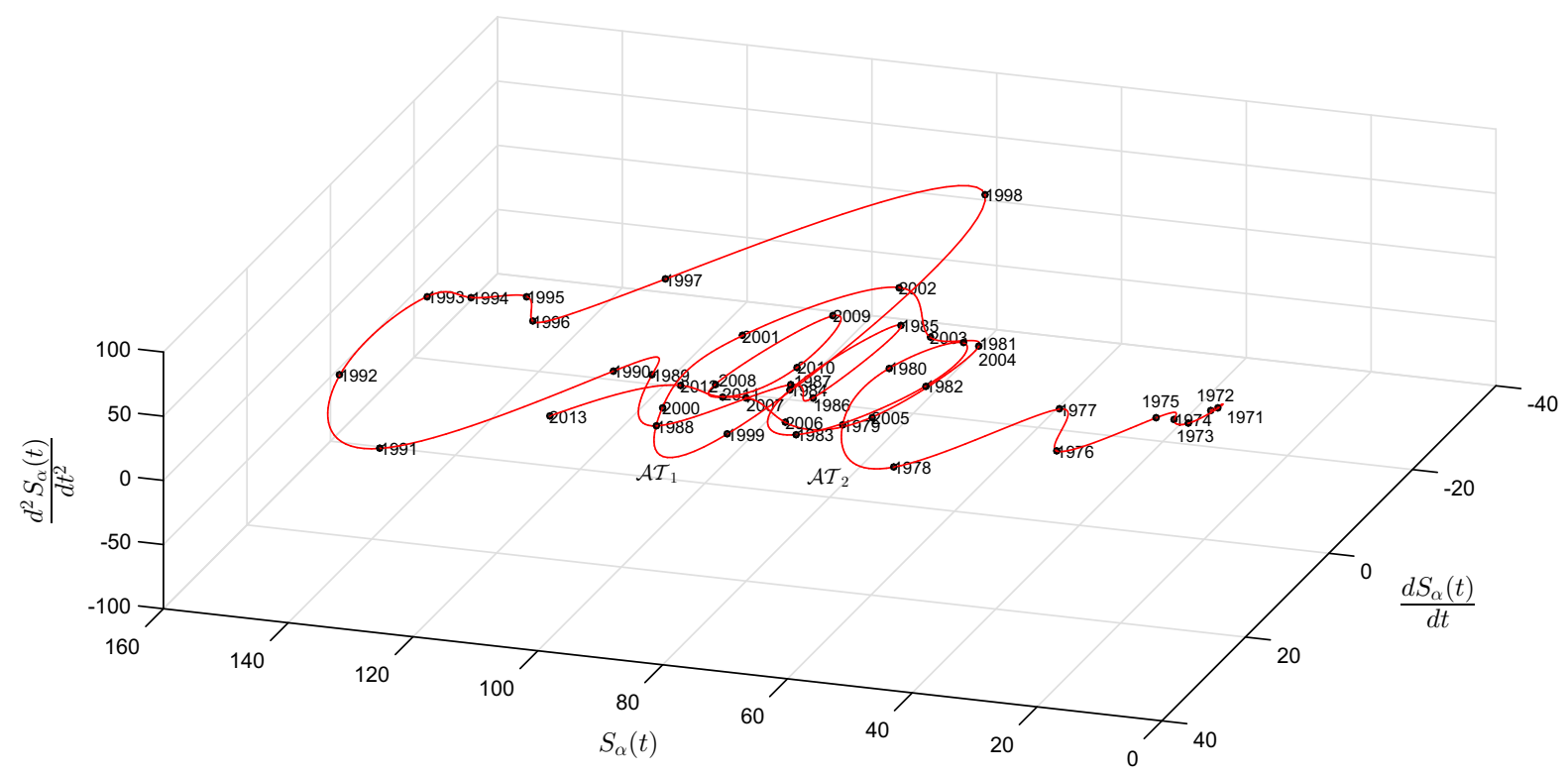

Fig. 6 The 3-dimensional SSP representation of $S_{\alpha}$ of global terrorism dynamics

are analyzing for the purpose of police control. The most famous case is, of course, the 11th September aircraft crash against the twin towers in New York, with the dramatic Guinness number of casualties in terrorist attacks. In bringing novelty and accuracy they can also explain dynamical jumps [52].

\section{Regional behavior of terrorism}

In this section we analyze terrorism dynamics in a per region basis. The GDT database divides Earth into twelve geographic regions, namely Australasia and Oceania, Central America and Caribbean, Central Asia, East Asia, Eastern Europe, Middle East and 
Fig. 7 The regions

$\mathcal{R}_{i}, i=1, \ldots, 8$, considered in the study

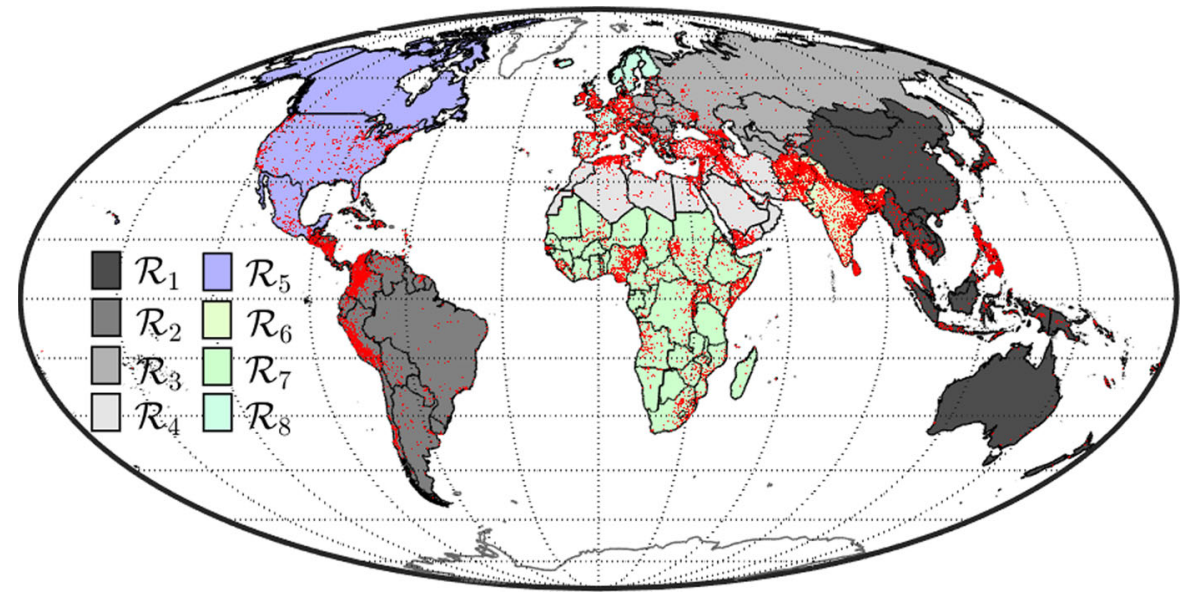

Fig. 8 Number of events per year, for regions $\mathcal{R}_{i}, i=1, \ldots, 8$, during the period 1970-2014

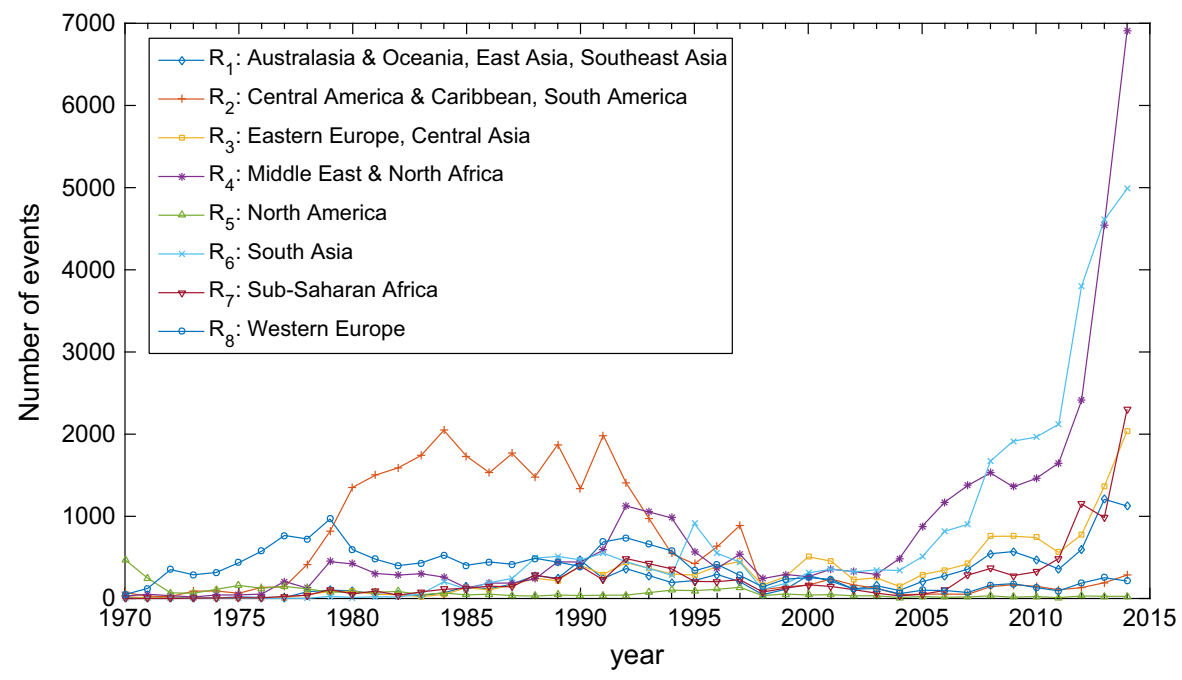

North Africa, North America, South America, South Asia, Southeast Asia, Sub-Saharan Africa and Western Europe. However, since for certain areas the number of events per year is small, we decided to merge some regions, obtaining eight distinct geographic zones: $\mathcal{R}_{1}=$ Australasia and Oceania, East Asia, Southeast Asia\}, $\mathcal{R}_{2}=$ \{Central America and Caribbean, South America $\}, \mathcal{R}_{3}=\{$ Eastern Europe, Central Asia $\}$, $\mathcal{R}_{4}=\{$ Middle East and North Africa $\}, \mathcal{R}_{5}=$ \{North America $\}, \mathcal{R}_{6}=\{$ South Asia $\}, \mathcal{R}_{7}=\{$ Sub-Saharan Africa $\}$ and $\mathcal{R}_{8}=$ \{Western Europe $\}$. In this way, we establish a compromise between preserving the original regionalization and using statistically significant data. Figures 7 and 8 depict the eight regions and the number of events per year for each $\mathcal{R}_{i}, i=1, \ldots, 8$, respectively.

In Figure 9 we depict the temporal evolution of the fractal dimension, $b$, and fractional entropy, $S_{\alpha}$
( $\alpha=0.7$ ), for regions $\mathcal{R}_{i}, i=1, \ldots, 8$. To improve readability we opt for not representing the BoltzmannGibbs-Shannon entropy, $S$.

We computed the 3-dimensional SSP maps, corresponding to the fractional entropy, $S_{\alpha}$, for all $\mathcal{R}_{i}$ regions considered. As illustrated in Fig. 10 for regions $\mathcal{R}_{2}=$ \{Central America and Caribbean, South America\} and $\mathcal{R}_{8}=\{$ Western Europe $\}$, most of the charts do not reveal such a clear dynamics as for the global case, namely the two attractors identified in the global dynamics are not visible in certain regions, as is the case of $\mathcal{R}_{8}$. Does it means that regional events are more irregular and so there is no synchronism? Or that the space sampling affects negatively the study of phenomena that have a global behavior?

According to Lugovskyy [18] terrorism has to do with economic conditions and inequality [22]. It decreases with population and education levels, but 
Fig. 9 Time evolution of the fractal dimension, $b$, and fractional entropy, $S_{\alpha}$ $(\alpha=0.7)$, for regions $\mathcal{R}_{i}, i=1, \ldots, 8$, during the period 1970-2014
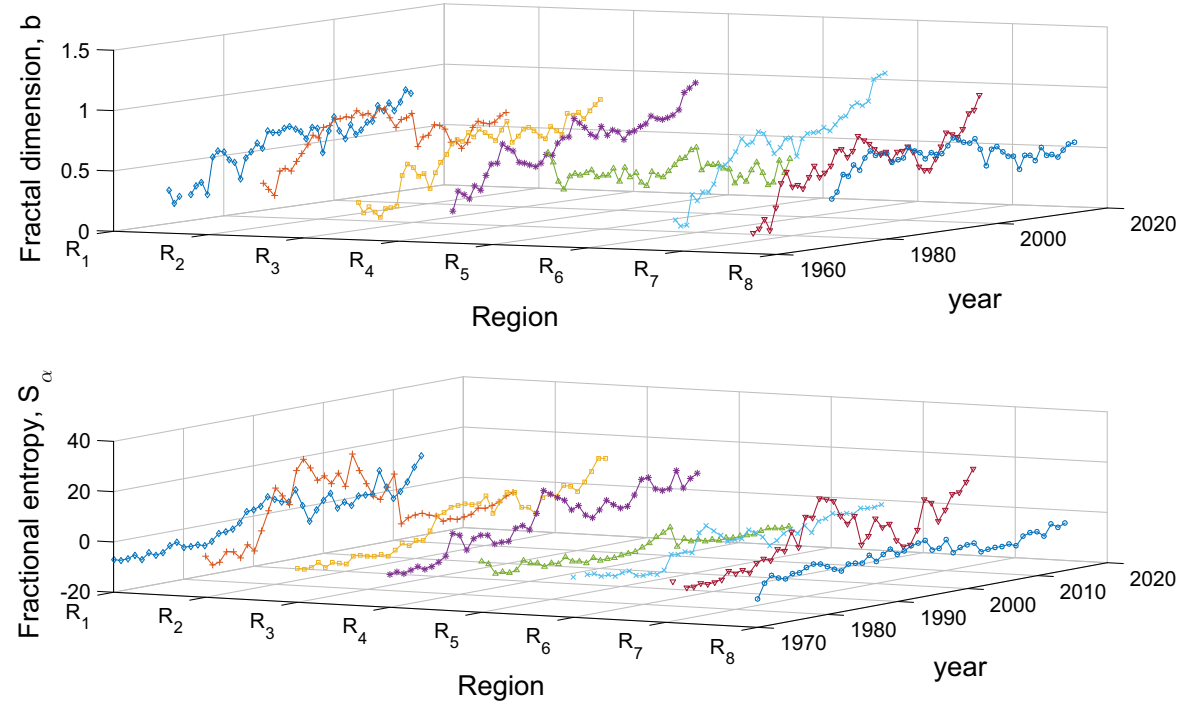

increases with a decrease in civil liberties [1]. According to other views, terrorism is a psychological process to lead some young people to follow a religion or ideology in calling for crime, violent terrorism acts, instead of communication, politics or warfare [4]. In a global perspective, instability, urban fearing and racism may support and feed terrorism simultaneously. Warring is an open conflict to address territorial expansion hopes, increased resources, improved welfare and political hegemony, having national heroes and future collective memories [13]. Terrorism is a veiled fight, made of anonymous actors, which is accompanied by negative forecasts on markets in the attacked societies, because it may precede local or regional open warring events, whenever victory cannot occur, or negotiated sharing power cannot take place [14]. Globalization may be hurt because of terrorist actions. Moving, voyages and transportation networks have been frequently attacked to obtain massive casualties in terrorist events (such as aircraft explosions). International trade, foreign direct investment and capital flows become conditioned under risk and uncertainty, paving the way to careful business strategies against negative environment, using emergency management to face market instability, and volatility [19]. All these direct and indirect costs mean increased negative spillover effects on the capitalist corporate economy. Terrorism countermeasures and vigilant attitude to promote security and avoid losses have high economic costs. Chemical attacks such as the sarin gas attack, in Tokyo (1995), promoted high worrying and economic costs with urban camera surveillance using closed-circuit television equipment in all public places, from airports to rail stations, trade centers and small units, theaters, bus stops and others) [53]. False alarms have the same threatening effects, obliging to stops, careful inspections, loss of time, delays and decreased productivity. Regional asymmetries may also be the result of different terrorism risk attacks [54]. Life under terrorism risk means inconvenience and is unpleasant [16]. Threat and terrorism expectations are detrimental factors for regional development and promotion [15].

\section{A complementary analysis of global terrorism}

In this section we adopt MDS and clustering tools to complement the analysis of global terrorism dynamics.

MDS is a technique for visualizing information in data $[17,29,30,34,59,62]$ that requires the definition of a similarity, or dissimilarity, index and the construction of an $s \times s$ matrix $\mathbf{C}$ of item-to-item similarities, or dissimilarities, where $s$ is the total number of items in an $r$-dimensional space. MDS extrapolates an approximate map in a $u$-dimensional space $(u<r)$ with the objects' represented as points. For low-dimensional spaces (e.g., $u=2$, or $u=3$ ) the resulting points can be displayed in a chart. The MDS interpretation is based on the emerging clusters and distances between points in the map. Thus, the units of the axes are meaningless, and we can rotate or translate the MDS map since the distances between points remain identical. 


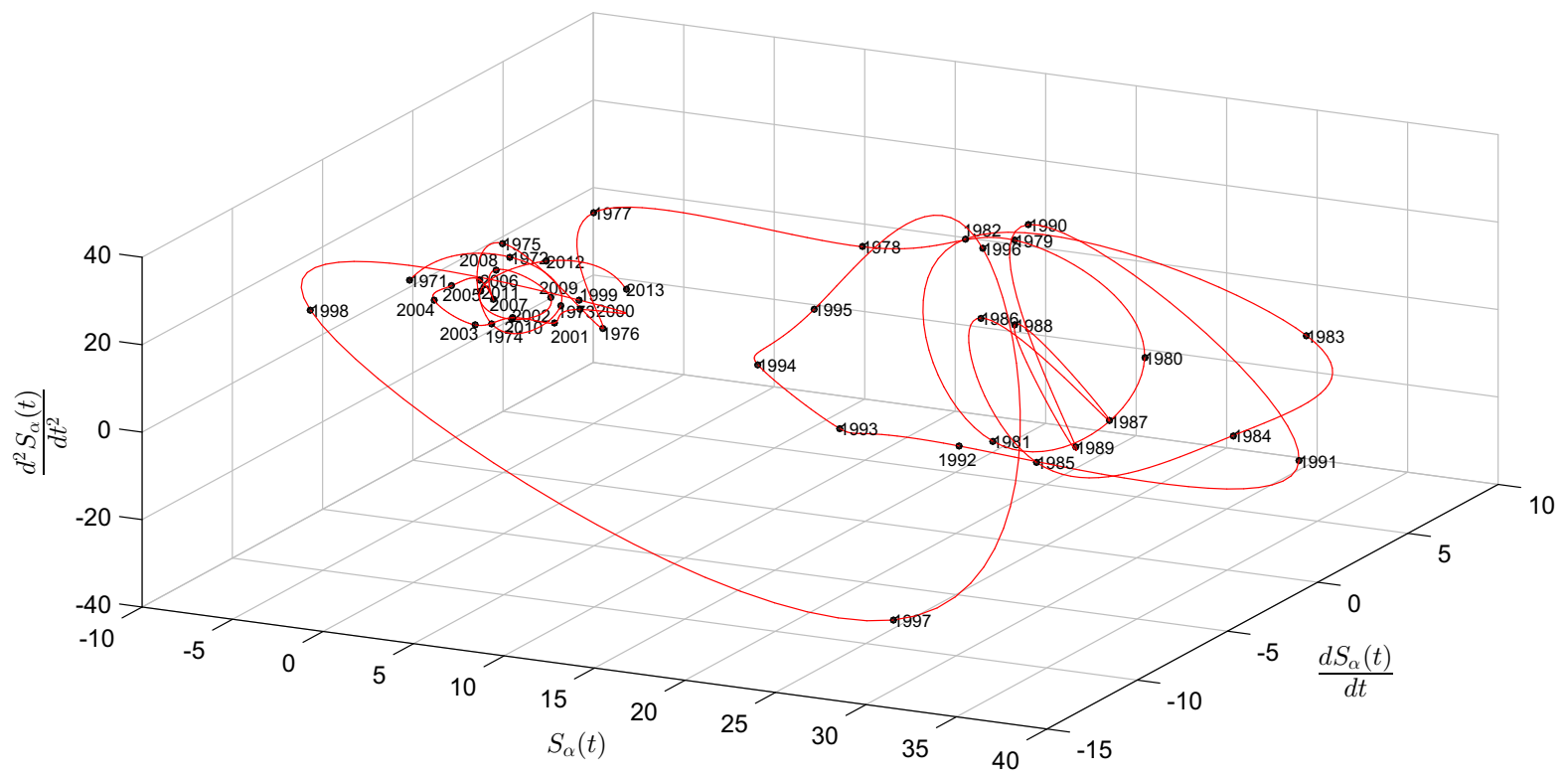

(a)

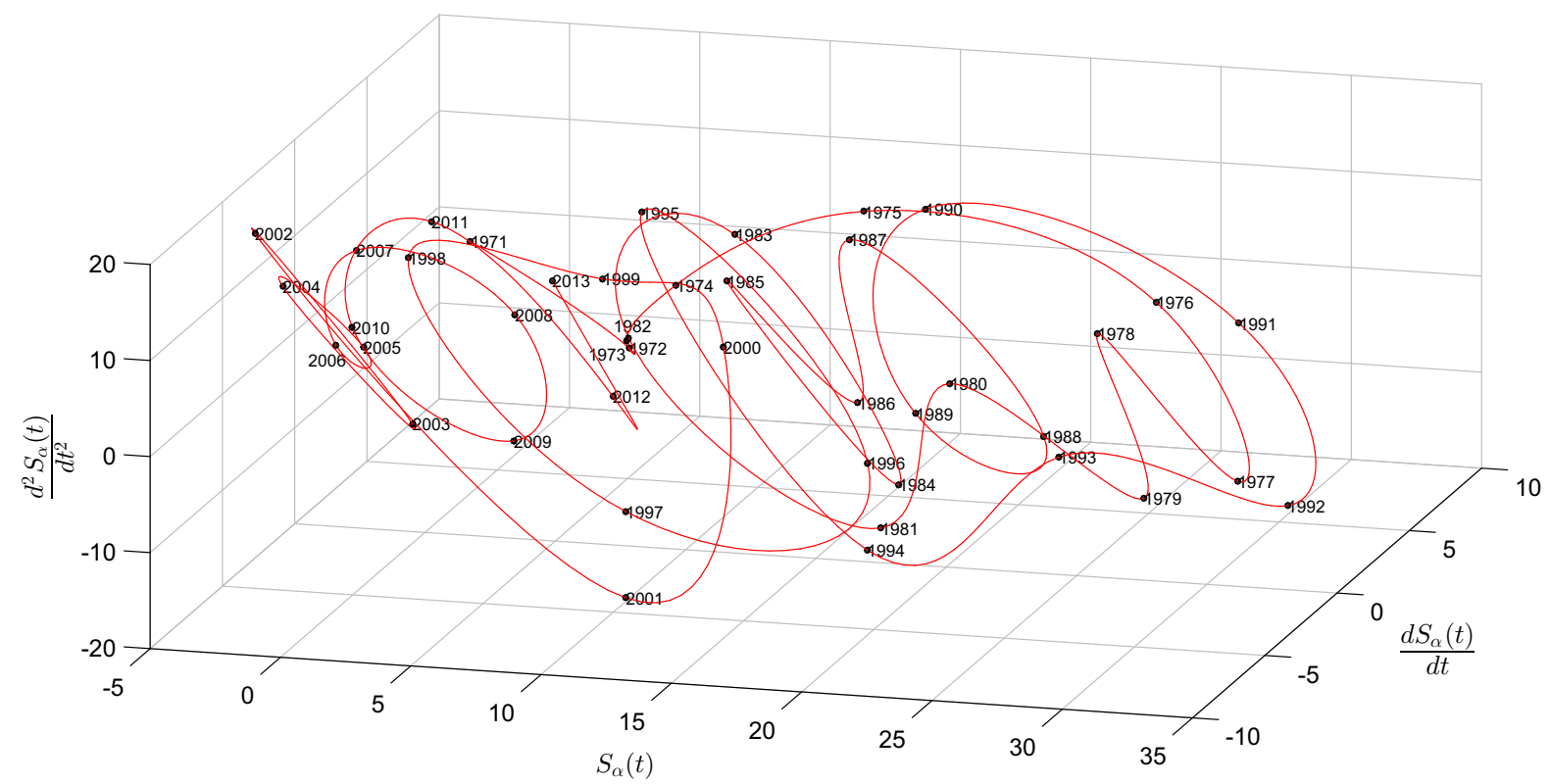

(b)

Fig. 10 The 3-dimensional SSP representation of $S_{\alpha}$, for regions: a $\mathcal{R}_{2} ; \mathbf{b} \mathcal{R}_{8}$

For each pair of years $\{P, Q\}=\{1970, \ldots, 2014\}$, we first calculate the spacial probability distribution of terrorist events, as shown in Sect. 3.2, and we compute the matrix $\mathbf{C}=\left[J S D_{\alpha}(P \| Q)\right]$, where $J S D_{\alpha}(P \| Q)(\alpha=0.7)$ represents the fractional
Jensen-Shannon divergence between the space probability distributions of years $\{P, Q\}$ :

$$
\begin{aligned}
& J S D_{\alpha}(P \| Q) \\
& =\frac{1}{2} \sum_{i} \sum_{j} p_{i j}\left\{\frac{p_{i j}^{-\alpha}}{\Gamma(\alpha+1)}\left[\ln p_{i j}+\psi(1)-\psi(1-\alpha)\right]\right\}
\end{aligned}
$$


Fig. 11 Tree generated by the $\mathrm{HC}$ algorithm, comparing the spacial statistical distributions of terrorist events, during the period 1970-2014, by means of the Jensen-Shannon divergence, $J S D_{\alpha}(P \| Q)$, $\alpha=0.7$

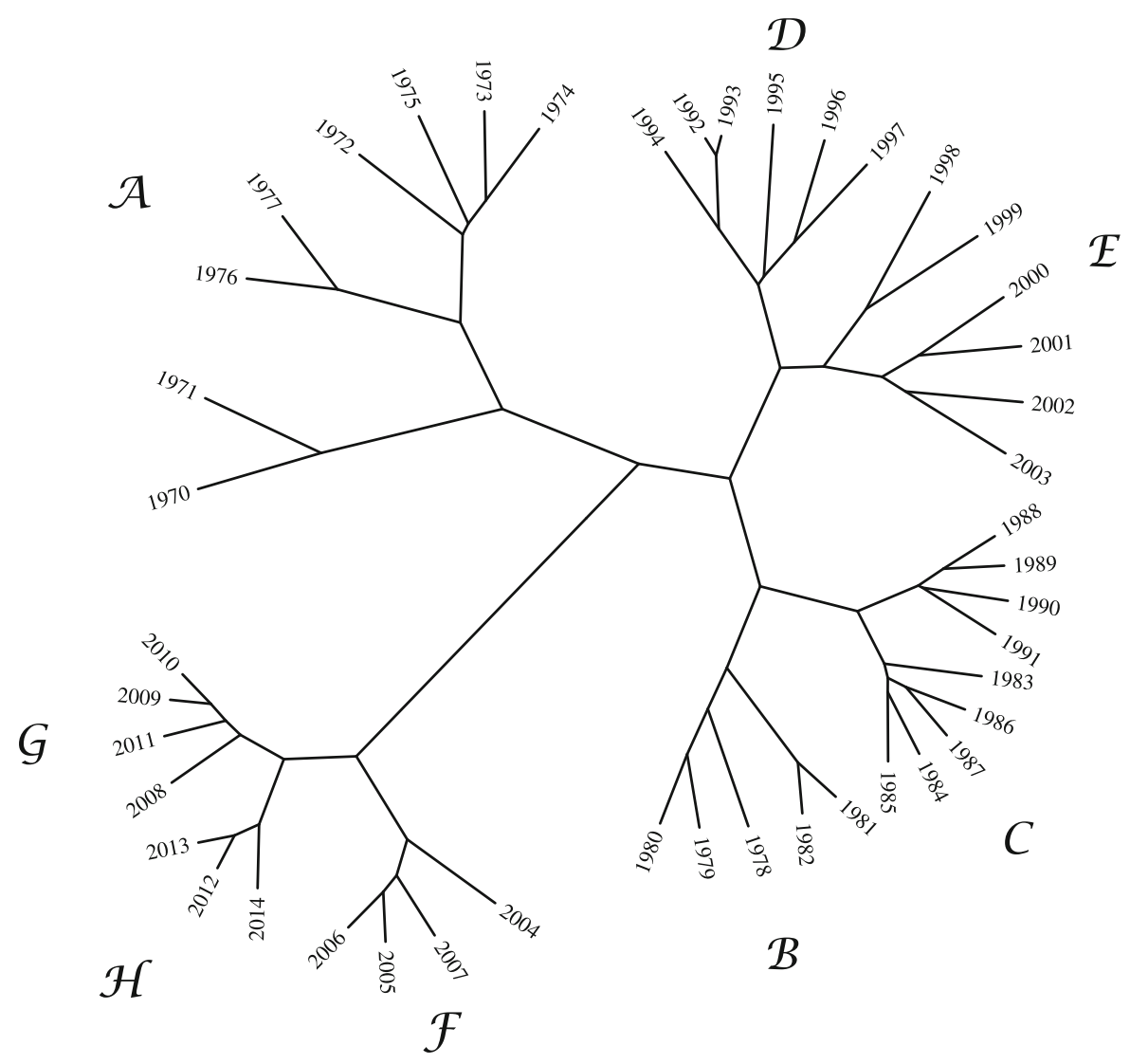

$$
\begin{aligned}
& +\frac{1}{2} \sum_{i} \sum_{j} q_{i j}\left\{\frac{q_{i j}^{-\alpha}}{\Gamma(\alpha+1)}\left[\ln q_{i j}+\psi(1)-\psi(1-\alpha)\right]\right\} \\
& -\sum_{i} \sum_{j} m_{i j}\left\{\frac{m_{i j}^{-\alpha}}{\Gamma(\alpha+1)}\left[\ln m_{i j}+\psi(1)-\psi(1-\alpha)\right]\right\},
\end{aligned}
$$

and $i \times j=36 \times 72$ is the size of the grid adopted for covering the Earth projection.

We pursuit the analysis by feeding with matrix $\mathbf{C}$ both a hierarchical clustering (HC) algorithm and the MDS, generating two alternative maps for visualizing the results.

Figure 11 depicts the tree generated by the $\mathrm{HC}$, based on the successive (agglomerative) clustering and average-linkage method. This representation is to be compared with that produced by the MDS.

The standard MDS analysis is based on the objects (interpreted as clusters) emerging in the final map. We can rely either in the direct visualization of the plot or in the implementation of some extra algorithm to extract the clusters. Here we consider an approach aimed to improve the visualization and the identification of patterns. The method allows the definition of more complex objects that generalize the usual simple clusters of points. We start by connecting the points that are closer in the MDS plot. This scheme produces several sets, $\mathcal{P}$, of interconnected points (nodes) that represent the building blocks of prototype complex objects. The resulting sets, $\mathcal{P}$, of interconnected points are compared by means of the distances between their nodes, and a new connection is established between those two nodes that are closer (i.e., between $\mathcal{P}_{i}$ and its neighbor $\mathcal{P}_{j}$ ). This scheme creates a second level of interconnection composed by more complex building blocks. The scheme is repeated iteratively until there is a continuous route interconnecting all points in the MDS, and no further processing is required. The interpretation of the MDS plot is now based not only on the clusters, but also on the structure of the interconnection between points that produce an $m$-dimensional pathway. 


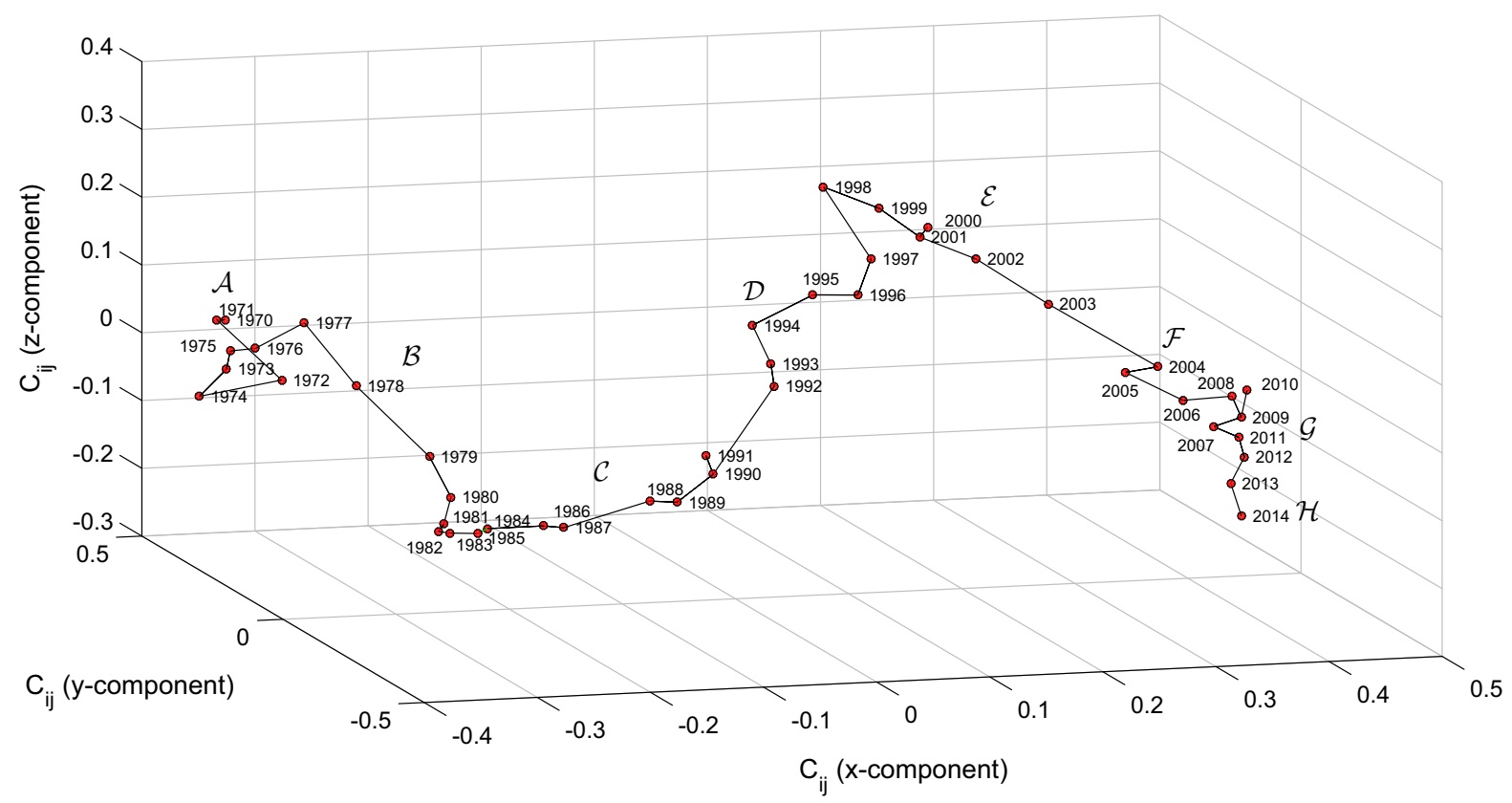

Fig. 12 Map generated by the MDS and the superimposed pathway, comparing the spacial statistical distributions of terrorist events, during the period 1970-2014, by means of the Jensen-Shannon divergence, $J S D_{\alpha}(P \| Q), \alpha=0.7$

Figure 12 represents the 3-dimensional MDS map and a superimposed pathway that has for nodes the $s$ MDS points. We can observe the emergence of the eight clusters identified previously in Fig. 4.

In conclusion, terrorism is a phenomenon reflecting many distinct conditions that change both in time and in space. The choice of mathematical and computational tools capable of handling the available data and revealing hidden aspects represents a formidable task. The results of several tools common in complex systems analysis provided a framework for scientific assessment of patterns and world regions (where the European Mid-East axis is one of the pieces of after cold-war times geopolitics). In fact, the fractal dimension, entropy, SSP and MDS lead to the same type of results and encourage further studies in the same line of thought.

\section{Conclusions}

This paper analyzed real-world data of terrorism attacks during the last 45 years. Three distinct mathematical tools were adopted to unveil the dynamics of these events along space and time.
Due to the plethora of causes (political, religious, economical, social or technological) and effects (number of deaths, injuries, magnitude of destructing effects or type of actions and weapons), the rigorous and quantified analysis of the information represents a considerable problem for an assertive analysis.

The proposed approach is, therefore, not yet a prediction tool for future events to occur, but provides a solid basis for further developments that can shed light for decreasing the trends of present-day dynamics.

Acknowledgments The authors acknowledge the National Consortium for the Study of Terrorism and Responses to Terrorism (START) for the Global Terrorism Database (http://www. start.umd.edu/gtd).

\section{References}

1. Abadie, A.: Poverty, political freedom, and the roots of terrorism. Am. Econ. Rev. 95, 50-56 (2005)

2. Abadie, A., Gardeazabal, J.: The economic costs of conflict: a case study of the Basque Country. Am. Econ. Rev. 93(1), 113-132 (2003)

3. Abe, S., Beck, C., Cohen, E.G.: Superstatistics, thermodynamics, and fluctuations. Phys. Rev. E 76(3), 031,102 (2007)

4. Alex, P.S.: Frameworks for conceptualising terrorism. Terror. Political Violence 16(2), 197-221 (2004) 
5. Asgarani, S., Mirza, B.: Two-parameter entropies, $s_{k, r}$, and their dualities. Phys. A Stat. Mech. Its Appl. 417, 185-192 (2015)

6. Baleanu, D., Diethelm, K., Scalas, E., Trujillo, J.J.: Models and Numerical Methods, vol. 3. World Scientific, Singapore (2012)

7. Bassil, C.: The effect of terrorism on tourism demand in the Middle East. Peace Econ. Peace Sci. Public Policy 20(4), 669-684 (2014)

8. Beck, C.: Generalised information and entropy measures in physics. Contemp. Phys. 50(4), 495-510 (2009)

9. Berrebi, C., Klor, E.F.: The impact of terrorism on the defence industry. Economica 77(307), 518-543 (2010)

10. Berry, M.V.: Diffractals. J. Phys. A Math. Gen. 12(6), 781797 (1979)

11. Bhatia, P.: On certainty and generalized information measures. Int. J. Contemp. Math. Sci. 5(21), 1035-1043 (2010)

12. Blomberg, S.B., Hess, G.D.: From (no) butter to guns? Understanding the economic role in transnational terrorism. Underst. Econ. Role Trans. Terror. (2006)

13. Burgoon, B.: On welfare and terror social welfare policies and political-economic roots of terrorism. J. Confl. Resolut. 50(2), 176-203 (2006)

14. Charles, A., Darné, O.: Large shocks and the September 11 th terrorist attacks on international stock markets. Econ. Modell. 23(4), 683-698 (2006)

15. Clauset, A., Woodard, R., et al.: Estimating the historical and future probabilities of large terrorist events. Ann. Appl. Stat. 7(4), 1838-1865 (2013)

16. Clauset, A., Young, M., Gleditsch, K.S.: On the frequency of severe terrorist events. J. Confl. Resolut. 51(1), 58-87 (2007)

17. Cox, T., Cox, M.: Multidimensional Scaling. Chapman \& Hall/CRC, Boca Raton (2001)

18. Lugovskyy, Cruz: J.: The economic determinants of terrorism. Int. J. Acad. Bus. World 9(1), 31-42 (2015)

19. Drakos, K.: Terrorism-induced structural shifts in financial risk: airline stocks in the aftermath of the September 11th terror attacks. Eur. J. Political Econ. 20(2), 435-446 (2004)

20. Esteban, M., Morales, B.: A summary on entropy statistics. Kybernetika-Praha 4(31), 337-346 (1995)

21. Fleckinger-Pelle, J., Lapidus, M.: Tambour fractal: vers une résolution de la conjecture de Weyl-Berry pour les valeurs propres du laplacien. Comptes rendus de l'Académie des sciences. Série 1, Mathématique 306(4), 171-175 (1988)

22. Goldstein, K.B.: Unemployment, inequality and terrorism: another look at the relationship between economics and terrorism. Undergrad. Econ. Rev. 1(1), 6 (2005)

23. Gupta, S., Clements, B., Bhattacharya, R., Chakravarti, S.: Fiscal consequences of armed conflict and terrorism in lowand middle-income countries. Eur. J. Political Econ. 20(2), 403-421 (2004)

24. Hanel, R., Thurner, S.: A comprehensive classification of complex statistical systems and an axiomatic derivation of their entropy and distribution functions. Europhys. Lett. 93(2), 20,006 (2011)

25. Ionescu, C.M.: The Human Respiratory System: An Analysis of the Interplay Between Anatomy, Structure, Breathing and Fractal Dynamics. Springer, Berlin (2013)
26. Kaniadakis, G.: Maximum entropy principle and power-law tailed distributions. Eur. Phys. J. B Condens. Matter Complex Syst. 70(1), 3-13 (2009)

27. Khinchin, A.I.: Mathematical Foundations of Information Theory, vol. 434. Courier Dover Publications, Mineola (1957)

28. Kilbas, A.A.A., Srivastava, H.M., Trujillo, J.J.: Theory and Applications of Fractional Differential Equations, vol. 204. Elsevier Science Limited, Amsterdam (2006)

29. Kruskal, J.: Multidimensional scaling by optimizing goodness of fit to a nonmetric hypothesis. Psychometrika 29(1), 1-27 (1964)

30. Kruskal, J., Wish, M.: Multidimensional Scaling. Sage Publications, Newbury Park (1978)

31. Lopes, A.M., Machado, J.: Fractional order models of leaves. J. Vib. Control 20(7), 998-1008 (2014)

32. Lopes, A.M., Machado, J., Pinto, C.M., Galhano, A.M.: Fractional dynamics and MDS visualization of earthquake phenomena. Comput. Math. Appl. 66(5), 647-658 (2013)

33. Lopes, A.M., Machado, J.T.: Dynamic analysis of earthquake phenomena by means of pseudo phase plane. Nonlinear Dyn. 74(4), 1191-1202 (2013)

34. Lopes, A.M., Machado, J.T.: Analysis of temperature timeseries: embedding dynamics into the mds method. Commun. Nonlinear Sci. Numer. Simul. 19(4), 851-871 (2014)

35. Lopes, A.M., Machado, J.T.: Integer and fractional-order entropy analysis of earthquake data series. Nonlinear Dyn. 84(1), 79-90 (2016)

36. Lopes, A.M., Machado, J.T.: Modeling vegetable fractals by means of fractional-order equations. J. Vib. Control (2015). doi:10.1177/1077546315581228

37. Luo, Y., Chen, Y.: Fractional Order Motion Controls. Wiley, Hoboken (2012)

38. Machado, J., Lopes, A., Duarte, F., Ortigueira, M., Rato, R.: Rhapsody in fractional. Fract. Calc. Appl. Anal. 17(4), 1188-1214 (2014)

39. Machado, J., Mata, M.E., Lopes, A.M.: Fractional state space analysis of economic systems. Entropy 17(8), 54025421 (2015)

40. Machado, J.A.T., Lopes, A.M.: Analysis and visualization of seismic data using mutual information. Entropy 15(9), 3892-3909 (2013)

41. Machado, J.T.: Fractional order generalized information. Entropy 16(4), 2350-2361 (2014)

42. Machado, J.T., Lopes, A.M.: The persistence of memory. Nonlinear Dyn. 79(1), 63-82 (2014)

43. Machado, J.T., Mata, M.E.: Pseudo phase plane and fractional calculus modeling of western global economic downturn. Commun. Nonlinear Sci. Numer. Simul. 22(1), 396406 (2015)

44. Mandelbrot, B.B.: How long is the coast of Britain? statistical self-similarity and fractional dimension. Science 156(3775), 636-638 (1967)

45. Mandelbrot, B.B.: The Fractal Geometry of Nature, vol. 173. Macmillan, New York (1983)

46. Miller, K.S., Ross, B.: An introduction to the fractional calculus and fractional differential equations. Wiley, New York (1993)

47. Newman, M.E.: Power laws, pareto distributions and zipf's law. Contemp. Phys. 46(5), 323-351 (2005) 
48. Pape, R.A.: The strategic logic of suicide terrorism. Am. Political Sci. Rev. 97(03), 343-361 (2003)

49. Pinto, C.M., Lopes, A.M., Machado, J.T.: A review of power laws in real life phenomena. Commun. Nonlinear Sci. Numer. Simul. 17(9), 3558-3578 (2012)

50. Pinto, C.M., Lopes, A.M., Machado, J.T.: Double power laws, fractals and self-similarity. Appl. Math. Modell. 38(15), 4019-4026 (2014)

51. Polderman, J.W., Willems, J.C.: Introduction to Mathematical Systems Theory: A Behavioral Approach, vol. 26. Springer, Berlin (1998)

52. Richardson, L.: What Terrorists Want: Understanding the Enemy, Containing the Threat. Random House Trade Paperbacks, New York City (2007)

53. Rose, A., Avetisyan, M., Chatterjee, S.: A framework for analyzing the economic tradeoffs between urban commerce and security against terrorism. Risk Anal. 34(8), 1554-1579 (2014)

54. Rose, A., Oladosu, G., Liao, S.Y.: Regional economic impacts of terrorist attacks on the electric power system of Los Angeles: a computable general disequilibrium analysis. In: Second Annual Symposium of the DHS Center for Risk and Economic Analysis of Terrorism Events, USC, Los Angeles, CA, August. Citeseer (2005)

55. Rrnyi, A.: On measures of entropy and information. Fourth Berkeley Symp. Math. Stat. Probab. 1, 547-561 (1961)

56. Samko, S.G., Kilbas, A.A., Marichev, O.I.: Fractional integrals and derivatives. Theory and Applications, Gordon and Breach, Yverdon (1993)

57. Schroeder, M.: Fractals, Chaos, Power Laws: Minutes from an Infinite Paradise. W.H. Freeman, New York (1991)
58. Sharma, B.D., Taneja, I.J.: Entropy of type $(\alpha, \beta)$ and other generalized measures in information theory. Metrika 22(1), 205-215 (1975)

59. Shepard, R.: The analysis of proximities: Multidimensional scaling with an unknown distance function. Psychometrika 27(I and II), 219-246 and 219-246 (1962)

60. Silva, M.F., Machado, J., Lopes, A.: Fractional order control of a hexapod robot. Nonlinear Dyn. 38(1-4), 417-433 (2004)

61. Tenreiro Machado, J., Pinto, C., Lopes, A.M.: Power law and entropy analysis of catastrophic phenomena. Math. Probl. Eng. 2013, 10 (2013). doi:10.1155/2013/562320

62. Torgerson, W.: Theory and Methods of Scaling. Wiley, New York (1958)

63. Tsallis, C.: Possible generalization of Boltzmann-Gibbs statistics. J. Stat. Phys. 52(1-2), 479-487 (1988)

64. Ubriaco, M.R.: Entropies based on fractional calculus. Phys. Lett. A 373(30), 2516-2519 (2009)

65. Valério, D., Trujillo, J.J., Rivero, M., Machado, J.T., Baleanu, D.: Fractional calculus: a survey of useful formulas. Eur. Phys. J. Spec.Top. 222, 1827-1846 (2013)

66. Wada, T., Suyari, H.: A two-parameter generalization of Shannon-Khinchin axioms and the uniqueness theorem. Phys. Lett. A 368(3), 199-205 (2007)

67. Weissman, S.H., Busch, K.G., Schouten, R.: Introduction to this issue: the evolution of terrorism from 1914 to 2014. Behav. Sci. Law 32(3), 259-262 (2014) 